\title{
On the Numerical Construction of Ellipsoidal Wave Functions
}

\author{
By F. M. Arscott, P. J. Taylor and R. V. M. Zahar
}

\begin{abstract}
The ellipsoidal wave equation, which is the most general equation derived by separation of the Helmholtz equation in confocal coordinates, presents unusual computational difficulties, and its solutions, despite their importance for physical applications, have not hitherto been effectively computed.

This paper describes a successful technique, which involves the solution of a four-term recursion and the simultaneous handling of two eigenparameters.
\end{abstract}

1. Introduction. The ellipsoidal wave equation, or Lamé wave equation, has been known in mathematical literature for a considerable time, certainly since 1926 when Ince [12] listed it among the equations derivable from the general second order linear differential equation with five regular singularities. More significantly, it is the equation which emerges when the technique of separation of variables is applied to the Helmholtz equation in ellipsoidal coordinates [3, 1.6]. Physically, therefore, its solution is a matter of considerable interest; there are applications, among others, to problems of scalar and electromagnetic scattering by an ellipsoid, and diffraction by an elliptic plate or through an elliptic aperture.

The equation has been the subject of some fairly extensive theoretical analysis, and quite a lot is known about the general properties of its solutions; e.g., [2]. Remarkably, however, computed results are almost wholly lacking, and indeed the usual computational techniques which serve for most other special functions are simply not applicable; this is because of mathematical difficulties to be mentioned shortly. The only numerical results-or formulae which can give such resultshitherto published are in the form of perturbation series or asymptotic series, corresponding roughly to low frequency and high frequency approximations, respectively [1], [7], [10]. The purpose of this paper is to report on a general method for computing the eigenvalues and the solutions.

There is an interesting parallel between this problem and the corresponding problem posed by Mathieu's equation; the latter was first formulated in 1868 and used in connection with an astronomical problem [15], [11]. Considerable progress was made regarding its theoretical solution, but it was not until some 60 years after Mathieu's original memoir that Ince accomplished the first systematic tabulation [13], [14]. For ellipsoidal wave functions, the corresponding gap has been similar; the greater problems of tabulation have been compensated by the availability of modern computing facilities. In fact, the difficulties are not so much computational as analytic; the limitation has been imposed not by any lack of speed or storage

Received October 6, 1981; revised March 19, 1982.

1980 Mathematics Subject Classification. Primary 65D20; Secondary 33A60, 65L15, 65Q05. 
facilities but simply by not knowing where to start. Indeed, for most of the calculations we shall describe, a relatively small computing aid like a COMMODORE PET or an HP97 will suffice; full-scale computer facilities are helpful but not essential.

2. The Ellipsoidal Wave Equation and Its Main Features. The ellipsoidal wave equation can be put into several forms, of which two are important for our present purpose and a third will be mentioned later, as it seems to offer an alternative line of attack.

(a) The 'Jacobian' form is

$$
\frac{d^{2} w}{d z^{2}}-\left(a+b k^{2} \operatorname{sn}^{2} z+q k^{4} \operatorname{sn}^{4} z\right) w=0,
$$

where the Jacobian elliptic function $\operatorname{sn} z=\operatorname{sn}(z, k)$ has modulus $k$.

A transformed version of this is important: we set

$$
z=K+i K^{\prime}-i z^{\prime}
$$

where $K, K^{\prime}$ are the usual quarter-periods, and obtain

$$
\frac{d^{2} w}{d z^{\prime 2}}-\left\{a^{\prime}+b^{\prime} k^{\prime 2} \operatorname{sn}^{2}\left(z^{\prime}, k^{\prime}\right)+q^{\prime} k^{\prime 4} \operatorname{sn}^{4}\left(z^{\prime}, k^{\prime}\right)\right\} w=0,
$$

where $k^{\prime}=\left(1-k^{2}\right)^{1 / 2}, a^{\prime}=-a-b-q, b^{\prime}=b+2 q, q^{\prime}=-q$.

(b) The 'algebraic' form, which is obtained from (2.1) by setting $t=\operatorname{sn}^{2} z$ is

$$
\begin{aligned}
t(t-1)(t-c) \frac{d^{2} w}{d t^{2}}+\frac{1}{2}\left\{3 t^{2}-2(1+c) t\right. & +c\} \frac{d w}{d t} \\
& +\left(\lambda+\mu t+\gamma t^{2}\right) w=0 .
\end{aligned}
$$

Here, for convenience, the parameters have been changed by setting

$$
k^{-2}=c, \quad a=-4 k^{2} \lambda, \quad b=-4 \mu, \quad q=-4 \gamma / k^{2} .
$$

The 'trigonometric' form will be given and discussed in Section 8 below.

Our objective is not the general solution of this equation but the construction of ellipsoidal wave functions, which are particular solutions characterized by boundary conditions or finiteness properties. These are given in the next section; now we pause to explain why this differential equation is both difficult and interesting.

(i) A rough classification of second order ordinary linear equations can be made on the basis of the number and type of their singularities. The 'hypergeometric type' comprises those equations which have precisely three regular singularities, or can be derived from such an equation as a special or confluent case, in which two or more singularities coalesce. This type includes the equations of Bessel, Legendre, Laguerre, Jacobi, and many others, whose properties are now quite extensively known.

The next level comprises equations of 'Heun type' which similarly are derivable from the general equation with four regular singularities. In this category fall the equations of Mathieu and Lamé, the spheroidal and paraboloidal wave equations, and others; very much less is known about such equations and their solutions.

The ellipsoidal wave equation, however, belongs to a higher type still, for in its algebraic form (2.4) it is seen to have three regular singularities (at $t=0,1$ and $c$ ) 
and an irregular singularity at $\infty$, which can only be obtained by confluence of two regular singularities. Thus (2.4) is a confluent case of the general equation with five regular singularities; it appears to be the first equation in this class to receive any detailed study.

(ii) The classical method of solution in series provides an excellent means of investigating differential equations of hypergeometric type, for such a formal solution can always be made to yield (possibly after some preliminary manipulation) a two-term recursion relation between successive coefficients, which is easily handled. For equations of Heun type, however, the recursion relation always involves at least three terms; such relations are far less tractable. It seems impossible to find series solutions in which the coefficients are given explicitly, and even their numerical computation proved-as already mentioned-beyond the power of 19th century mathematics.

The situation is still worse with regard to the ellipsoidal wave equation, because series solutions are found always to involve at least a four-term recursion relation between the coefficients, so that even the rather involved methods which succeed for Heun type equations cannot be directly used.

Another way of regarding this phenomenon is to say that the method of solution in series replaces a second order differential equation problem by a difference equation problem. In the case of hypergeometric type functions, the difference equation is first order; for Heun type equations it is of second order, but for the ellipsoidal wave equation it is of third order - thus, in principle, a harder problem than the original.

(iii) For functions of hypergeometric type, great use is made of expressions for solutions as definite or contour integrals. For functions of Heun type, no such solutions have been found; instead, solutions can be shown to satisfy relatively simple Fredholm integral equations, and these are useful in investigation of properties of solutions. For the ellipsoidal wave equation, however, there are no corresponding simple integral equations, and we have only certain 'quadratic' integral equations or two-dimensional linear equations.

(iv) Finally, the ellipsoidal wave equation is a 'two-parameter' problem. In most applications of (2.1) or (2.4) the parameters $q$ and $k$ (or $\gamma$ and $c$ ) are known or have to be regarded as known; the former has a physical meaning such as frequency while the latter is a geometrical parameter. By contrast, the parameters $a, b$ (or $\lambda, \mu$ ) are not physically significant, indeed they arise only from the process of separation. Our problem, generally, is to determine eigenvalues of these parameters, so that nontrivial solutions of the equation exist which satisfy the appropriate side conditions.

Two-parameter problems are not unknown in connection with simpler equations, but in such problems one can generally dispose of the unknown parameters one at a time. No such device seems to be possible for the ellipsoidal wave equation; the two eigenparameters have to be handled as a pair.

3. Ellipsoidal Wave Functions. Ellipsoidal wave functions are, by definition, solutions of (2.1) which are doubly-periodic (with periods $4 K, 4 i K^{\prime}$ ) or, equivalently, solutions of (2.4) which are finite at the three finite singularities $t=0,1$ and $c$. 
It is known [2] that these fall into eight types; as solutions of (2.1) they take the forms

$$
(\operatorname{sn} z)^{\rho}(\operatorname{cn} z)^{\sigma}(\operatorname{dn} z)^{\tau} F(z),
$$

where $\rho, \sigma, \tau=0$ or 1 and $F(z)$ is even, doubly-periodic with periods $2 K, 2 i K^{\prime}$, and having singularities only at $i K^{\prime}$ and congruent points, hence an integral function of $\mathrm{sn}^{2} z$. As solutions of (2.4) they take the form

$$
t^{\rho / 2}(t-1)^{\sigma / 2}(t-c)^{\tau / 2} G(t),
$$

where $G(t)$ is an integral function of $t$.

However, the computational problems are essentially the same for all the eight types of functions, so we shall discuss only the simplest type, that for which $\rho=\sigma=\tau=0$. The modifications needed to deal with the remaining types will be given in Section 9, where the standard notation for these functions is also given.

The eigenvalues $a, b$ (or $\lambda, \mu$ ) and corresponding eigenfunctions are known to form a 'triangularly infinite' set; for technical reasons they are denoted by

$$
a_{2 n}^{m}, b_{2 n}^{m}, \operatorname{uel}_{2 n}^{m}(z), \quad\left(\lambda_{2 n}^{m}, \text { etc. }\right)
$$

or, more fully if necessary,

$$
a_{2 n}^{m}(q, k), b_{2 n}^{m}(q, k), \operatorname{uel}_{2 n}^{m}(z, q, k) \quad\left(\lambda_{2 n}^{m}(\gamma, c), \text { etc. }\right)
$$

where $m=0,1, \ldots, n ; n=0,1, \ldots$ to infinity. The indices $n, m$ are related to the zeros of the eigenfunctions; $\operatorname{uel}_{2 n}^{m}(z)$ has precisely $m$ zeros on the segment $(0, K)$ and $(n-m)$ zeros on $\left(K, K+i K^{\prime}\right)$; as a function of $t, \operatorname{uel}_{2 n}^{m}$ has $m$ zeros on $t \in(0,1)$ and $(n-m)$ zeros on $(1, c)$; these zeros are all simple.

4. Known Numerical Information. (a) When $q=0(\gamma=0)$, the eigenvalues of $b$ (or $\mu)$ are given by

$$
b=2 n(2 n+1), \quad \mu=-n\left(n+\frac{1}{2}\right),
$$

and, for given $n$, the eigenvalues of $a$ (or $\lambda$ ) are the roots of an algebraic equation of order $n+1$; thus, for $q=0(\gamma=0)$ the eigenvalues of $b(\mu)$ are $(n+1)$-fold, but the eigenvalues of $a(\lambda)$ are easily shown to be all simple. Regarded as an eigenvalue-pair, then, each pair $(a, b)$ (or $(\lambda, \mu))$ is simple.

(b) For the lowest eigenvalue-pair, a perturbation solution is known, that is, series for $a_{0}^{0}(q), b_{0}^{0}(q)$ and uel $_{0}^{0}(q)$ proceeding in powers of $q$; reference [1] gives these series to $O\left(q^{2}\right)$. For the next two eigenvalues of this type, the series have been computed only to $O(q)$ and higher terms seem impossible to calculate explicitly; for higher eigenvalues, perturbation series have not been calculated at all.

(c) However, asymptotic series for large $|q|$ are given in [7], valid for all eigenvalues; these provide a useful comparison with the eigenvalues computed by the method to be described in this paper, and in some cases yield starting-points for the iterative calculation.

5. The Computational Procedure. Since we are seeking a solution of the form (3.1) or (3.2) with $\rho=\sigma=t=0$, we may assume $F(z)$ in the form of a series in $\operatorname{sn}^{2} z$, or $G(t)$ as a series in $t$. For definiteness, we shall deal with Eq. (2.4) henceforth, since the manipulations do not involve the relatively unfamiliar formulae for elliptic functions. 
Assuming the formal solution of (2.4) as

$$
w=\sum_{0}^{\infty} \alpha_{r} t^{r},
$$

we obtain by substitution in (2.4) the recurrence system

$$
\begin{gathered}
\lambda \alpha_{0}+\frac{1}{2} c \alpha_{1}=0 \\
\mu \alpha_{0}+\{\lambda-(1+c)\} \alpha_{1}+3 c \alpha_{2}=0 \\
\gamma \alpha_{r}+\{\mu+(r+3 / 2)(r+1)\} \alpha_{r+1} \\
+\left\{\lambda-(1+c)(r+2)^{2}\right\} \alpha_{r+2}+c(r+5 / 2)(r+3) \alpha_{r+3}=0, \quad r \geqslant 0
\end{gathered}
$$

(If we adopt the convention that $\alpha_{r}=0$ for $r<0$, then (5.2a, b) agree with (5.3) for $r=-2,-1$.) We also choose to normalize the solution $w$ so that $\alpha_{0}=1$.

By applying the classical theory of linear difference equations to the third order equation (5.3), it can be shown that it possesses three types of solutions: those with $\alpha_{r+1} / \alpha_{r}$ asymptotic, as $r \rightarrow \infty$, to $1,1 / c$ and $-\gamma / r^{2}$, respectively. We recall, however, that $w$ is to be an integral function of $t$, so that the series in (5.1) has an infinite radius of convergence. Hence $w$ must correspond to the solution of (5.3) for which $\alpha_{r+1} / \alpha_{r} \sim-\gamma / r^{2}$ as $r \rightarrow \infty$. This means that in comparison with the other solutions of (5.3), the sequence for $\left\{\alpha_{r}\right\}$ associated with $w$ is minimal (see Gautschi [9]) and that its computation by forward recurrence will be numerically unstable. However, it can be verified that the sufficient conditions stated in [16] for the convergence and numerical stability of backward recurrence are satisfied, so that for fixed $\gamma$ and $\mu$ the sequence $\left\{\alpha_{r}\right\}$ can be computed by Miller's algorithm. That is, if for fixed $\lambda$ and $\mu$ we define the function of $N, y_{r}(N)$, by

$$
\begin{gathered}
x_{N}=1, \quad x_{N+1}=x_{N+2}=0, \\
x_{r}=-1 / \gamma\left[\{\mu+(r+3 / 2)(r+1)\} x_{r+1}\right. \\
\left.+\left\{\lambda-(1+c)(r+2)^{2}\right\} x_{r+2}+c(r+5 / 2)(r+3) x_{r+3}\right], \\
\quad r=N-1, N-2, \ldots, 0, \\
y_{r}(N)=x_{r} / x_{0}, \quad r=0,1,2, \ldots,
\end{gathered}
$$

then the $y_{r}(N)$ are estimates of $\alpha_{r}$ in the sense that

$$
\lim _{N \rightarrow \infty} y_{r}(N)=\alpha_{r}
$$

Therefore, for any given $\lambda$ and $\mu$, one can in principle compute the required solution $\left\{\alpha_{r}\right\}$ of (5.3) to any desired accuracy by taking $N$ sufficiently large. As a consequence, $(5.2 \mathrm{a}, \mathrm{b})$ can be regarded as a system of algebraic equations for $\lambda$ and $\mu$ alone:

$$
\begin{gathered}
F(\lambda, \mu, \gamma)=\lambda \alpha_{0}+\frac{1}{2} c \alpha_{1}=0, \\
G(\lambda, \mu, \gamma)=\mu \alpha_{0}+\{\lambda-(1+c)\} \alpha_{1}+3 c \alpha_{2}=0,
\end{gathered}
$$

and an iterative method can be employed to compute its roots. (The dependence of $F, G$ on $\gamma$ arises, of course, from the dependence of $\alpha_{0}, \alpha_{1}, \alpha_{2}$ on $\gamma$.) 
Thus, the basic computational procedure can be described as follows:

(i) A trial pair of values $\lambda^{(0)}, \mu^{(0)}$ is chosen and $i$ is set to 0 ;

(ii) A moderately large value of $N$ is taken and estimates of $\alpha_{r}^{(i)}$ are calculated by scheme (5.4);

(iii) Eqs. (5.5) and an iterative rule are then used to obtain improved estimates $\lambda^{(i+1)}, \mu^{(i+1)}$; step (ii) is then repeated with $i$ replaced by $i+1$ until a stage is reached when $\lambda^{(i)}, \mu^{(i)}$ and $\lambda^{(i+1)}, \mu^{(i+1)}$ agree to within the required accuracy.

In addition to (a) the selection of an iterative method for step (ii), two details in the above programme of operations remain to be settled: (b) the technique for choosing initial values $\lambda^{(0)}, \mu^{(0)}$, and (c) the value $N$ to be taken in (5.4). We shall discuss each of these details in turn.

(a) The simplest iterative method for improving the values of $\lambda$ and $\mu$ is that of successive substitution, because once the $\alpha_{r}^{(i)}$ are known, system (5.5) can be solved directly for the new $\lambda^{(i+1)}, \mu^{(i+1)}$. In this problem, however, the method may not enjoy the property of local convergence, and even if it does converge, it may do so extremely slowly. Better possibilities are offered by the two-dimensional secant method or by Newton's method. We choose to employ Newton's method because local convergence is assured, and because the amount of work per step is less than that in the secant method if the computations are suitably organized. Thus, we set

$$
\lambda^{(i+1)}=\lambda^{(i)}+\delta \lambda^{(i)}, \quad \mu^{(i+1)}=\mu^{(i)}+\delta \mu^{(i)},
$$

where $\delta \lambda^{(i)}, \delta \mu^{(i)}$ are given by

$$
\begin{aligned}
& \delta \lambda^{(i)} F_{\lambda}\left(\lambda^{(i)}, \mu^{(i)}, \gamma\right)+\delta \mu^{(i)} F_{\mu}\left(\lambda^{(i)}, \mu^{(i)}, \gamma\right)+F\left(\lambda^{(i)}, \mu^{(i)}, \gamma\right)=0 \\
& \delta \lambda^{(i)} G_{\lambda}\left(\lambda^{(i)}, \mu^{(i)}, \gamma\right)+\delta \mu^{(i)} G_{\mu}\left(\lambda^{(i)}, \mu^{(i)}, \gamma\right)+G\left(\lambda^{(i)}, \mu^{(i)}, \gamma\right)=0 .
\end{aligned}
$$

In order to compute the derivatives of $F, G$ with respect to $\lambda, \mu$, one needs to compute the derivatives $p_{r}=\partial \alpha_{r} / \partial \lambda$ and $q_{r}=\partial \alpha_{r} / \partial \mu$. By differentiation of (5.3), one finds that $p_{r}$ and $q_{r}$ satisfy the inhomogeneous recurrence relations

$$
\begin{aligned}
\gamma p_{r}+\{\mu & +(r+3 / 2)(r+1)\} p_{r+1} \\
& +\left\{\lambda-(1+c)(r+2)^{2}\right\} p_{r+2}+c(r+5 / 2)(r+3) p_{r+3}=-\alpha_{r+2} \\
\gamma q_{r}+\{\mu & +(r+3 / 2)(r+1)\} q_{r+1} \\
& +\left\{\lambda-(1+c)(r+2)^{2}\right\} q_{r+2}+c(r+5 / 2)(r+3) q_{r+3}=-\alpha_{r+1}
\end{aligned}
$$

It can be demonstrated that the $p_{r}$ and $q_{r}$ associated with $w$ are the minimal solutions of (5.7a) and (5.7b), respectively. Further, their values $p_{r}^{(i)}$ and $q_{r}^{(i)}$ at any stage of the iteration can be computed in a numerically stable manner by backward recurrence using the inhomogeneous form of Miller's algorithm; that is, we solve (5.7a), (5.7b) for $r=N-1, N-2, \ldots, 1$ starting with the values $p_{r}=q_{r}=0$ for $r=N, N+1$, $N+2$.

(b) The second computational issue to be resolved is that of finding sufficiently good starting values $\lambda^{(0)}$ and $\mu^{(0)}$ for the procedure to work; because of the number and relative closeness of the eigenvalue-pairs it is necessary to have quite close estimates. Our procedure has been to compute along a pair of eigenvalue curves, starting from the values for $\gamma=0$. In this case, one sees from (5.3) that when $\mu$ has the special value $-n\left(n+\frac{1}{2}\right)$, the series can be truncated, so that $\alpha_{r}=0$ for 
$r \geqslant n+1$. The condition on $\lambda$ is that the determinant of the first $(n+1)$ equations must vanish, i.e., an equation of degree $(n+1)$. Although it is not apparent from (5.2) and (5.3), the roots of the equation are all real, distinct and positive. These give starting points for the computation of the eigenvalue curves $\lambda(\gamma)$, which are obtained by taking $\mu=-n\left(n+\frac{1}{2}\right), n=0,1,2, \ldots$, and $\lambda=\lambda_{n}^{m}, m=0,1, \ldots, n$, as the zeros in increasing order of the determinantal equation. (For the particular values $c^{-1}=0.1,0.3,0.5,0.7,0.9$ and for $n \leqslant 30$, these are derived easily from [5], with $\lambda=\frac{1}{4} \mathrm{ch}$ in the notation of that reference.)

An alternative starting point for such plotting of pairs of eigenvalue curves is provided by the asymptotic formulae for $\lambda, \mu$, which can be easily obtained from the results in [7], and which are translated to the notation of this paper in Section 7.

Once the values of $\lambda(\gamma), \mu(\gamma)$ have been computed for a given value of $\gamma$, a first order continuity procedure can be employed to estimate the values corresponding to an augmented $\gamma+\Delta \gamma$. That is, we define

$$
\lambda(\gamma+\Delta \gamma)=\lambda(\gamma)+\Delta \lambda, \quad \mu(\gamma+\Delta \gamma)=\mu(\gamma)+\Delta \mu
$$

where $\Delta \lambda, \Delta \mu$ are given by

$$
\begin{aligned}
& \Delta \lambda F_{\lambda}(\lambda(\gamma), \mu(\gamma), \gamma)+\Delta \mu F_{\mu}(\lambda(\gamma), \mu(\gamma), \gamma) \\
& +\Delta \gamma F_{\gamma}(\lambda(\gamma), \mu(\gamma), \gamma)=0 \\
& \text { (5.8b) } \Delta \lambda G_{\lambda}(\lambda(\gamma), \mu(\gamma), \gamma)+\Delta \mu G_{\mu}(\lambda(\gamma), \mu(\gamma), \gamma) \\
& +\Delta \gamma G_{\gamma}(\lambda(\gamma), \mu(\gamma), \gamma)=0 .
\end{aligned}
$$

The derivatives $s_{r}=\partial \alpha_{r} / \partial \gamma$ required in these expressions satisfy the recurrence relation

$$
\begin{aligned}
\gamma s_{r}+\{\mu+(r+3 / 2)(r+1)\} s_{r+1}+\{\lambda & \left.-(1+c)(r+2)^{2}\right\} s_{r+2} \\
& +c(r+5 / 2)(r+3) s_{r+3}=-\alpha_{r}
\end{aligned}
$$

and, as before, can be computed by backward recurrence starting with $s_{r}=0$ for $r=N, N+1, N+2$.

(c) The final computational detail to be discussed is the choice of $N$ to be taken in (5.4). By straightforward algebra, it can be shown, as in [16], that the relative error $E$ in the computation of $\alpha_{r}$ satisfies

$$
E \sim\left|\frac{\alpha_{N+1}}{\alpha_{r}} \cdot \frac{\beta_{r}}{\beta_{N+1}}\right|
$$

as $N \rightarrow \infty$, where $\beta_{r}$ is the solution of (5.3) for which $\beta_{r+1} / \beta_{r} \sim 1 / c$ when $|c|>1$. Consequently, an estimate for the required $N$ can be obtained by replacing each expression in (5.10) by its asymptotic form (assuming that both $N$ and $r$ are large enough). Thus, an approximate value is the smallest $N$ for which

$$
\left(\frac{(r-1) !}{N !}\right)^{2}|\gamma c|^{N-r+1}<\frac{1}{2} \cdot 10^{-\alpha},
$$

where $\alpha$ is the desired number of significant decimal figures.

6. Some Observations on the Computational Procedure. In the course of carrying out the computations described in Section 5 , it proved necessary at certain points to take a larger value of $N$ than expected, or to make an exceptionally good choice of $\lambda$ 
and $\mu$. The phenomenon is due, apparently, to instability in the general recursion formula (5.3) which arises for certain critical values of $\lambda, \mu, \gamma, c$ in the following manner:

[A similar difficulty arises in connection with the three-term recursion involved in the computation of Mathieu functions, and is discussed in [6]: the situation in the present case is qualitatively similar but quantitatively much harder to analyze.]

Let us write

$$
v_{r}=\frac{\alpha_{r+1}}{\alpha_{r}}
$$

Then, as we remarked in Section 5 , the asymptotic behavior of $v_{r}$, as $r \rightarrow \infty$, is

$$
v_{r} \sim 1, \frac{1}{c} \text { or }-\gamma r^{-2}
$$

our computational procedure is designed to pick out the minimal solution and follow it backwards.

The recursion (5.3) may be written

$$
A_{r} v_{r} v_{r-1} v_{r-2}+B_{r} v_{r-1} v_{r-2}+C_{r} v_{r-2}+\gamma=0,
$$

where $A_{r}=c\left(r+\frac{1}{2}\right)(r+1), B_{r}=\lambda-(1+c) r^{2}, C_{r}=\mu+\left(r-\frac{1}{2}\right)(r-1)$. If $v_{r}$ were (locally) approximately constant, say $v_{r}=v$, then (6.2) would become

$$
A_{r} v^{3}+B_{r} v^{2}+C_{r} v+\gamma=0
$$

which is, of course, the characteristic equation of the difference equation (5.3) assuming constant coefficients. In general, we can expect the backward recursion process to be stable so long as the two numerically smaller roots of (6.3) are significantly different in modulus so that the minimal solution should not be contaminated by the next solution to it.

For $r$ large, the equation (6.3) becomes

$$
c v^{3}-(1+c) v^{2}+v=0
$$

with roots $1, c^{-1}, 0$. It is easy to see that, as $r$ decreases from $\infty$, the roots of (6.3) originally at 0 and $c^{-1}$ each move to the left and so may eventually become about equal in magnitude with opposite signs. Clearly one situation in which this may occur is when $C_{r} \simeq 0$, i.e., $\mu \simeq-\left(r-\frac{1}{2}\right)(r-1)$. For the ranges of values of $\lambda, \mu, \gamma$ occurring in the graphs shown, it is indeed found that the computational process becomes unduly sensitive just for such values of $\mu$, namely $0,-1.5,-5,-10.5$, $-18,-27.5, \ldots$ That is not to say, of course, that sensitivity can be expected only at such points, but experimentally these are the only places where it occurs in the computations here described.

One method of avoiding this sensitivity is to adapt the simple device of 'centering', analogous to that described in [6]. That is to say, we choose an integer $M<N$, take $\alpha_{N}=1, \alpha_{N+1}=\alpha_{N+2}=0$, and use (5.3) with $r=N-1, N-2, \ldots, M$ to compute the $\alpha_{r}$ back to $\alpha_{M}$. We then use (5.2a, b) and (5.3) with $r=0,1, \ldots, M-3$, leaving $\alpha_{0}$ temporarily unspecified; this gives the coefficients $\alpha_{0}$ to $\alpha_{M}$ determined up to a constant factor; then we choose this factor so that the two values of $\alpha_{M}$ agree. We are then left with the equations (5.3) for which $r=M-2$ and $r=M-1$, so far unused; since we have now computed all the coefficients $\alpha_{r}$, these two equations can be solved to give the next values of $\lambda$ and $\mu$. This process can be designated as 'centered' on the integer $M$; the procedure described in Section 5 is, of course, 
centered on $M=0$. Although only approximate, this method of centering has proved to be adequate for the eigenvalues discussed in this article.

The necessity of choosing exceptionally good values of $\lambda$ and $\mu$ become more marked as $n$ and $m$ increase, and numerical tests indicated that this difficulty is, for the most part, independent of the choice of $N$, if $N$ is large enough. Indeed, it appears that the local regions of convergence of Newton's method simply become smaller as $n$ and $m$ increase. It seems advisable, therefore, as mentioned in [17], to modify the recurrences by injecting another parameter into the numerical procedure. For this purpose, we choose to express the formal solution (5.1) about the generic point $t_{0}$ in the complex plane

$$
w=\sum_{0}^{\infty} \alpha_{r}\left(t-t_{0}\right)^{r}
$$

thus obtaining, in place of (5.2), (5.3), the recurrence system

$$
A_{r} \alpha_{r}+B_{r} \alpha_{r+1}+C_{r} \alpha_{r+2}+D_{r} \alpha_{r+3}+E_{r} \alpha_{r+4}=0, \quad r \geqslant 0
$$

where

$$
\begin{aligned}
& A_{r}=2 \gamma, \\
& B_{r}=2\left(2 \gamma t_{0}+\mu\right)+(2 r+3)(r+1), \\
& C_{r}=2\left(\gamma t_{0}^{2}+\mu t_{0}+\lambda\right)+2(r+2)^{2}\left(3 t_{0}-1-c\right), \\
& D_{r}=\left(3 t_{0}^{2}-2(1+c) t_{0}+c\right)(r+3)(2 r+5), \\
& E_{r}=2 t_{0}\left(t_{0}-1\left(t_{0}-c\right)(r+3)(r+4) .\right.
\end{aligned}
$$

(The first two relations in the recurrence are merely (6.6) with $r=-2,-1$, taking $\alpha_{-2}=\alpha_{-1}=0$.)

Upon experimentation, it was found that with $t_{0}=1$, and the other values of the parameters quoted here, the Newton method converged without further difficulty. (This change corresponds, in fact to assuming a solution of (2.1) in the form of a series containing even powers of $\mathrm{cn} z$ rather than of $\mathrm{sn} z$.)

7. Results. As an indication of the results* computed by the method described in Sections 5 and 6, we attach Figures 1 and 2 showing the first six eigenvalue curves in the case $c=2$, that is to say, for the pairs $(n, m)=(0,0),(1,0),(1,1)$, $(2,0),(2,1),(2,2)$. In the computations, $\Delta \gamma=\frac{1}{4}$ was used throughout.

The corresponding values for $\gamma=0$ are

$\begin{array}{cccc}n & m & \lambda & \mu \\ 0 & 0 & 0 & 0 \\ 1 & 0 & \frac{1}{2}(3-\sqrt{3})=0.6340 & -1.5 \\ 1 & 1 & \frac{1}{2}(3+\sqrt{3})=2.3660 & -1.5 \\ 2 & 0 & (5-\sqrt{13})=1.3944 & -5 \\ 2 & 1 & 5 & -5 \\ 2 & 2 & (5+\sqrt{13})=8.6054 & -5\end{array}$

* Note. In private communication with the first author, Professor L. Fox of the Oxford University Computing Laboratory has indicated that a two-parameter shooting method, using matrix techniques, yields results which agree with those of the authors. He observed similar difficulties to ours in the sensitive areas mentioned above. 
The asymptotic series are as follows:

(a) As $\gamma \rightarrow \infty$

$$
\lambda \sim A \sqrt{\gamma}-B+O\left(\gamma^{-1 / 2}\right), \quad \mu \sim-\gamma+C \sqrt{\gamma}+D+O\left(\gamma^{-1 / 2}\right),
$$

where

$$
\begin{array}{ll}
A=2 \sqrt{2} b, & B=\frac{1}{2}\left(b^{2}-4 \sqrt{2} a b+9 / 16\right), \\
C=2(b \sqrt{2}+a), & D=\frac{1}{2} a^{2}+\frac{1}{2} b^{2}+3 / 16,
\end{array}
$$

with $a, b$ depending on the integers $n, m$ :

$$
a=n-m+\frac{1}{4}, \quad b=m+\frac{1}{4} .
$$

(b) For the case $\gamma \rightarrow-\infty$, we set $\gamma^{\prime}=-\gamma$, then as $\gamma^{\prime} \rightarrow \infty$,

$$
\begin{aligned}
& \lambda=-2 \gamma^{\prime}+A^{*} \sqrt{\gamma^{\prime}}-B^{*}+O\left(\gamma^{\prime-1 / 2}\right), \\
& \mu \sim 3 \gamma^{\prime}-C^{*} \sqrt{\gamma^{\prime}}+D^{*}+O\left(\gamma^{\prime-1 / 2}\right),
\end{aligned}
$$

where

$$
\begin{array}{ll}
A^{*}=4 b+2 \sqrt{2} a, & B^{*}=\frac{1}{2} a^{2}+b^{2}+2 \sqrt{2} a b+3 / 32, \\
C^{*}=2(a \sqrt{2}+b), & D^{*}=\frac{1}{4}\left(a^{2}+b^{2}\right)+3 / 16
\end{array}
$$

$a, b$ being as in (7.2).

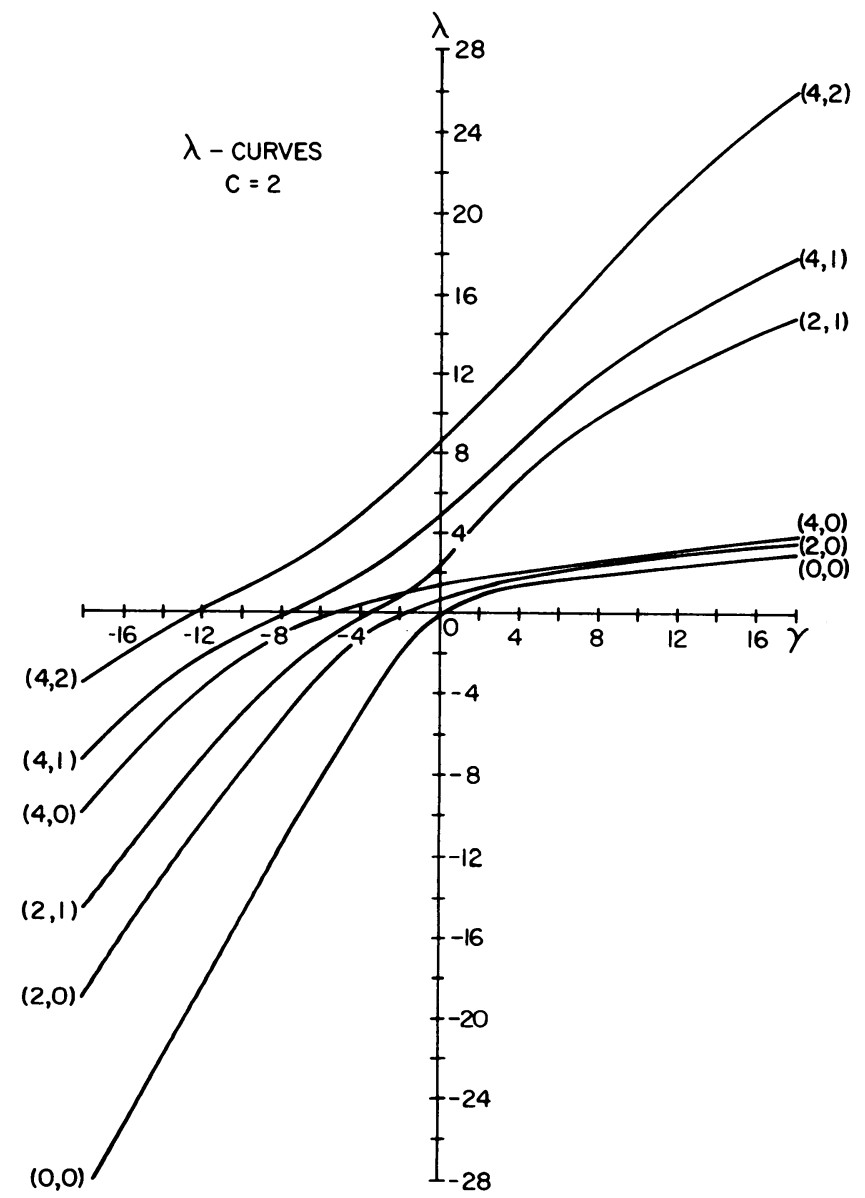

Figure 1 


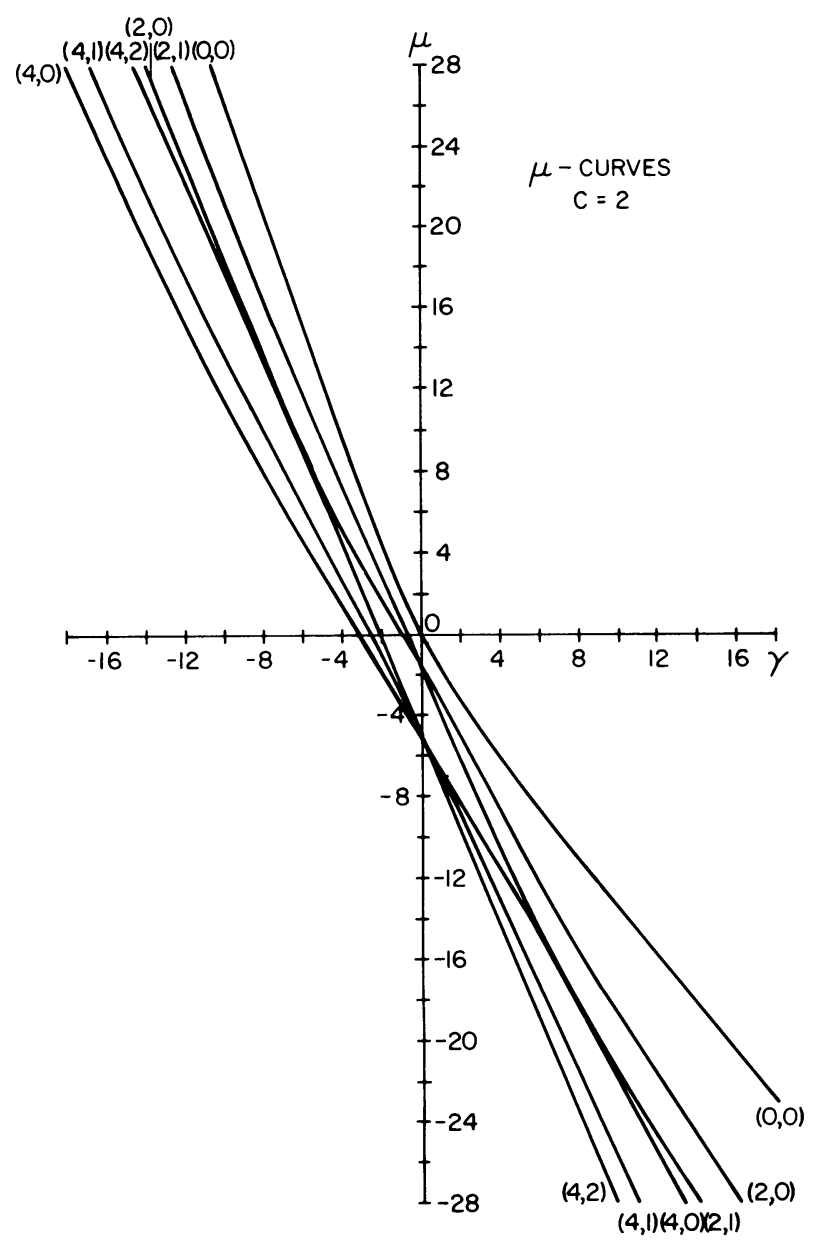

Figure 2

[Note. In the diagrams, the eigenvalue curves have been numbered to match the corresponding ellipsoidal wave functions $\operatorname{uel}_{0}^{0}, \operatorname{uel}_{2}^{0}, \operatorname{uel}_{2}^{1}, \operatorname{uel}_{4}^{0}, \operatorname{uel}_{4}^{1}, \operatorname{uel}_{4}^{2}$, the lower index being $2 n$ rather than $n$.]

8. Other Forms of the Equation; Solution by Trigonometric Series or Neumann Series. An interesting version of the ellipsoidal wave equation, and one which may prove computationally useful, is obtained by putting

$$
\operatorname{am} z=v \Leftrightarrow \operatorname{sn} z=\sin v \Leftrightarrow t=\sin ^{2} v
$$

in (2.1), (2.4), giving the "trigonometric form"

$$
\begin{aligned}
\left(1-k^{2} \sin ^{2} v\right) \frac{d^{2} w}{d v^{2}}-k^{2} \sin v \cos v \frac{d w}{d v} & \\
& -\left(a+b k^{2} \sin ^{2} v+q k^{4} \sin ^{4} v\right) w=0
\end{aligned}
$$

or, using multiple angles,

$$
\begin{aligned}
\left(1-\frac{1}{2} k^{2}+\frac{1}{2} k^{2} \cos 2 v\right) \frac{d^{2} w}{d v^{2}} & -\frac{1}{2} k^{2} \sin 2 v \frac{d w}{d v} \\
& +(R+S \cos 2 v+T \cos 4 v) w=0
\end{aligned}
$$


where

$$
-R=a+\frac{1}{2} b k^{2}+q k^{4}, \quad S=\frac{1}{2} k^{2}\left(b+q k^{2}\right), \quad T=\frac{-q k^{4}}{8} .
$$

We now seek to solve this by a trigonometric series; the form which corresponds to a uel-function is a series of even cosines

$$
w=\frac{1}{2} c_{0}+\sum_{1}^{\infty} c_{r} \cos 2 r v .
$$

Substitution in (8.3) yields a five-term recursion:

$$
\begin{gathered}
R c_{0}+\left(S-k^{2}\right) c_{1}+T c_{2}=0 \\
\frac{1}{2} S c_{0}+\left(\frac{1}{2} T+R-4\left(1-\frac{1}{2} k^{2}\right)\right) c_{1}+\left(\frac{1}{2} S-3 k^{2}\right) c_{2}+\frac{1}{2} T c_{3}=0 \\
\frac{1}{2} T c_{r-2}+\left[\frac{1}{2} S-(r-1)\left(r-\frac{1}{2}\right) k^{2}\right] c_{r-1}+\left[R-4 r^{2}\left(1-\frac{1}{2} k^{2}\right)\right] c_{r} \\
+\left[\frac{1}{2} S-(r+1)\left(r+\frac{1}{2}\right) k^{2}\right] c_{r+1}+\frac{1}{2} T c_{r+2}=0, \quad r \geqslant 2
\end{gathered}
$$

The fact that we have five terms instead of four in the recursion may be compensated by the symmetries in $(8.5 \mathrm{c})$. These series were first considered by Campbell [8].

A similar analysis to that of Section 5 shows that, as $r \rightarrow \infty$, the ratio $c_{r+1} / c_{r}$ must have one of the asymptotic forms

$$
\frac{2 k^{2}}{T} r^{2}, \quad-\frac{1+k^{\prime}}{1-k^{\prime}}, \quad-\frac{1-k^{\prime}}{1+k^{\prime}}, \quad \frac{T}{2 k^{2}} r^{-2},
$$

and clearly the numerical treatment must be such as to pick out the solution which has the last-mentioned behavior.

Mention should also be made of another possible mode of solution, namely the use of Neumann series (i.e., series of Bessel functions). Such series again lead to five-term recursion relations; details are given in [4].

9. The Other Types of Ellipsoidal Wave Function. The exposition in this paper has concentrated on only one of the eight types of ellipsoidal wave functions, but in fact the other seven types seem to be amenable to the same treatment.

Referring to (3.1) and (3.2), the eight types are distinguished by the eight combinations of values 0,1 taken by $\rho, \sigma, \tau$. The standard notation [2], [3] is to use the general symbol el $(z)$ and prefix the letters $\mathrm{s}, \mathrm{c}, \mathrm{d}$ according to the occurrence of the extraneous factors $\mathrm{sn} z, \mathrm{cn} z, \mathrm{dn} z$ in the representation (3.1) (or $\mathrm{u}$, for unity, if there are none such). Thus the eight types are written uel, sel, cel, del, scel, sdel, cdel, scdel, with suitable suffixes.

For the purposes of this paper it is more convenient to take the solutions in the form (3.1). By tedious working we find that the differential equation to be satisfied by $G(t)$ has the form

$$
\begin{aligned}
t(t-1)(t-c) G^{\prime \prime}(t)+ & \frac{1}{2}\left(A_{2} t^{2}-2 A_{1} t+A_{0}\right) G^{\prime}(t) \\
& +\left(\lambda-\lambda_{0}+\left(\mu+\mu_{0}\right) t+\gamma t^{2}\right) G(t)=0,
\end{aligned}
$$


where the values of $A_{2}, A_{1}, A_{0}, \lambda_{0}, \mu_{0}$ are as follows

$\begin{array}{ccccccccc}\text { Function type } & \rho & \sigma & \tau & A_{2} & A_{1} & A_{0} & \lambda_{0} & \mu_{0} \\ \text { uel } & 0 & 0 & 0 & 3 & 1+c & c & 0 & 0 \\ \text { sel } & 1 & 0 & 0 & 5 & 2+2 c & 3 c & \frac{1}{4}(1+c) & \frac{1}{2} \\ \text { cel } & 0 & 1 & 0 & 5 & 1+2 c & c & \frac{1}{4} c & \frac{1}{2} \\ \text { del } & 0 & 0 & 1 & 5 & 2+c & c & \frac{1}{4} & \frac{1}{2} \\ \text { scel } & 1 & 1 & 0 & 7 & 2+3 c & 3 c & \frac{1}{4}+c & \frac{3}{2} \\ \text { sdel } & 1 & 0 & 1 & 7 & 3+2 c & 3 c & 1+\frac{1}{4} c & \frac{3}{2} \\ \text { cdel } & 0 & 1 & 1 & 7 & 2+2 c & c & \frac{1}{4}(1+c) & \frac{3}{2} \\ \text { scdel } & 1 & 1 & 1 & 9 & 3+3 c & 3 c & 1+c & 3\end{array}$

Specifically

$$
\begin{aligned}
A_{2} & =2(\rho+\sigma+\tau)+3, \\
A_{1} & =(1+\rho)(1+c)+\tau+\sigma c, \\
A_{0} & =(2 \rho+1) c, \\
4 \lambda_{0} & =(\rho+\tau)^{2}+(\rho+\sigma)^{2} c, \\
4 \mu_{0} & =(\rho+\sigma+\tau)(\rho+\sigma+\tau+1) .
\end{aligned}
$$

When Eq. (9.1) is solved formally by a series

$$
G(t)=\sum_{0}^{\infty} \alpha_{r} t^{r},
$$

then the recursion (corresponding to (5.3)) is

$$
\begin{aligned}
& \gamma \alpha_{r}+\left[\mu+\mu_{0}+(r+1)\left(r+\frac{1}{2} A_{2}\right)\right] \alpha_{r+1} \\
& +\left[\lambda-\lambda_{0}-(r+2)\left(A_{1}+(r+1)(1+c)\right)\right] \alpha_{r+2} \\
& +(r+3)\left[(r+2) c+\frac{1}{2} A_{0}\right] \alpha_{r+3}=0 .
\end{aligned}
$$

Grateful acknowledgement is made to the Natural Sciences and Engineering Research Council of Canada for assistance to the authors through research grants. The authors' thanks are also expressed to the referee for a number of helpful comments.

Department of Applied Mathematics

University of Manitoba

Winnipeg, Manitoba, Canada R3T 2N2

Department of Mathematics

University of Stirling

Stirling, Scotland

Département d'Informatique et de Recherche Opérationelle

Université de Montréal

C. P. 6128, Succersale A

Montréal, P. Q., Canada H3C 3J7 
1. F. M. ARSCOTr, "Perturbation solutions of the ellipsoidal wave equation," Quart. J. Math., v. 7, 1956, pp. $161-174$.

2. F. M. ARSCOTt, "A new treatment of the ellipsoidal wave equation," Proc. London Math. Soc., v. 33, 1959, pp. 21-50.

3. F. M. Arscott, Periodic Differential Equations, Pergamon Press, New York, 1964.

4. F. M. Arscotr, "Neumann-series solutions of the ellipsoidal wave equation," Proc. Roy. Soc. Edinburgh, Sect A, v. 67, 1965, pp. 69-77.

5. F. M. Arscott \& I. M. Khabaza, Tables of Lamé Polynomials, Pergamon Press, New York, 1962.

6. F. M. ARscott, R. Lacroix \& E. T. ShymansKi, "A three-term recursion and the computation of Mathieu functions," Proc. Eighth Manitoba Conf. on Numerical Mathematics, 1978, pp. 107-115.

7. F. M. ARSCOTt \& B. D SLEEman "High-frequency approximations to ellipsoidal wave functions," Mathematika, v. 17, 1970, pp. 39-46.

8. R. Campbell, "Sur la vibration d'un haut-parleur elliptique," C. R. Acad. Sci. Paris, v. 288, 1949, pp. 970-972.

9. W. GautschI, “Zur Numerik rekurrenter Relationen,” Computing v. 9, 1972, pp. 107-126.

10. B. A. Hargrave \& B. D. Sleeman, "Uniform asymptotic expansions for ellipsoidal wave functions," J. Inst. Math. Appl., v. 14, 1974, pp. 31-40.

11. G. W. Hill, "On the part of the motion of the lunar perigee," Acta. Math., v. 8, 1886, pp. 1-36.

12. E. L. INCE, Ordinary Differential Equations, Dover (Longman's), 1926.

13. E. L. INCE, “Tables of the elliptic-cylinder functions," Proc. Roy. Soc. Edinburgh, v. 52, 1932, pp. 335-423.

14. E. L. INCE, "Zeros and turning-points of the elliptic-cylinder functions," Proc. Roy. Soc. Edinburgh, v. 52, 1932, pp. 424-433.

15. E. Mathieu, "Mémoire sur le mouvement vibratoire d'une membrane de forme elliptique," $J$. Math. Pures Appl., v. 13, 1868, pp. 137-203.

16. R. V. M. ZAhAR, “A mathematical analysis of Miller's algorithm,” Numer. Math., v. 27, 1977, pp. 427-447.

17. R. V. M. ZaHAR, "Recurrence techniques for a differential eigenvalue problem," Proc. Eighth Manitoba Conf. on Numerical Mathematics, 1978, pp. 479-485. 